\section{Effects of studying a subset of familiar items on recall of the remaining items: The John Brown effect*}

\author{
MICHAEL A. KARCHMER $†$ and EUGENE WINOGRAD \\ Emory University, Atlanta, Ga. 30322
}

Prior to attempting recall of the 50 U.S. states, two experimental groups studied subsets of 25 states each while the control group read unrelated material. The experimental groups differed in that one group studied the 25 states most easily recalled, as determined by norms, and the other group studied the 25 most difficult states. Interference was found for the states not studied, replicating Brown's (1968) findings. In Experiment 2, Ss were required to recall the studied states before recalling those not studied. Again, recall of the subset not studied was poorer for the experimental groups. Output interference was implicated in discussing the findings.

Brown (1968) has reported data that suggest that the recall of a very familiar set of items such as the 50 U.S. states may be interfered with when the interpolated task is, in fact, studying half of the set itself. In Brown's first experiment, one group studied a list of 25 states and then attempted to recall all 50 states. The interesting finding was that recall of the 25 states not studied, called the "nonlist" states by Brown, suffered in comparison to a control group which had not studied any states at all. Since it seems highly unlikely that such familiar items as "Minnesota" or "Oregon" were erased from storage while other state names were being studied, the phenomenon is of interest as an instance of output or retrieval interference.

The first study reported here essentially attempted to replicate Brown's first study with an additional manipulation. The preexperimental accessibility of the subset studied by the experimental group prior to recal of all 50 states was varied on the basis of independently obtained norms of state recall.

\section{EXPERIMENT 1 \\ Method}

First, norms for state recall were obtained. Eighty-five paid volunteers attending the Emory summer session were tested in groups and allowed 5 min to write down as many of the 50 states as they could. The states were then ranked according to the number of Ss listing them. To give an insight into the collective cognitive map of these $\mathrm{Ss}$, the five highest

* This work was supported by a National Institute of Mental Health predoctoral fellowship to the first author and by Grant GB-18703 from the National Science Foundation to the second author.

+ Now at St. Francis Xavier University (Sydney campus), Sydney, N.S.. Canada. ranking states were California Georgia, New York, North Carolina, and South Carolina, while the five lowest ranking states were Wisconsin, Delaware, Iowa, Wyoming, and Minnesota. The mean number of states listed was 41.89 . The correlation between frequency of recall of a state and its rank when recalled was - 59 . A median split was made between the 25 states most frequently recalled, the high subset, and the 25 states least recalled, the low subset. Mean recall was 23.43 states for the high subset and 18.46 states for the low subset.

There were three groups of Ss in the experiment proper. The $S s$ in the experimental groups were given prepared sheets on which one of the subsets of 25 states was listed. Group PH was exposed to the high subset, and Group PL was given the low subset. Two unsystematically ordered lists were prepared of each subset and used about equally often for each group. These Ss were told that they were to spend the next 5 min studying the list of states so that they could remember them. They were instructed that the order in which the states were listed was of no importance. The $S s$ in the control group, Group C, were allowed $5 \mathrm{~min}$ to read a story about the laying of a cable under the sea. Following the first task, all Ss were allowed $5 \mathrm{~min}$ to write as many of the 50 state names that they could. They were told to write them in any order they wished and were allowed to abbreviate. They were also asked to draw a line under the state they had last written as $E$ announced each elapsed minute. The Ss were 82 Emory undergraduates fulfilling a requirement of the introductory psychology course; they were assigned randomly to groups. Only data from American-born Ss were used. There were $26 \mathrm{Ss}$ in
Group PH, 29 Ss in Group PL, and 27 Ss in Group C. All Ss were tested individually.

\section{RESULTS}

The mean numbers of high and low states recalled by each group is shown in Table 1, along with standard deviations. The critical comparisons concern recall of the subset not studied, the nonlist states. Recall of nonlist states for the experimental groups was compared with the corresponding subset for the control group, after Brown, by individual $t$ tests. For Group PL, recall of the high subset was significantly poorer than was Group $C$ recall of the high subset, $\mathrm{t}(54)=2.09, \mathrm{p}<.05$. For Group PH, recall of the nonlist low subset was lower, but not significantly so, than recall for Group $\mathrm{C}, t=1.31$. In view of the outcomes of the $t$ tests, it was decided to perform a 2 by 2 analysis of variance on the nonlist recall data, treating the four conditions as independent, in spite of the fact that both means from the control group are from the same Ss. ${ }^{1}$ This analysis shows significant main effects for treatment (experimental vs control $), \quad F(1,105)=5.41, \quad p<.05$, and for recall subset, $F(1,105)=84.28, \quad p<.01$, with no interaction, $F<1$. We conclude that the evidence, on the whole, supports Brown's (1968) findings that studying part of a well-learned set may interfere with recall of the rest of the set.

Output curves on which recall of nonlist states was plotted cumulatively by minutes showed a constant difference over the recall period favoring the control group in each case. There was no indication of convergence, a finding also reported by Brown.

One interpretation of the John Brown effect is to attribute it to output or retrieval interference, i.e., Ss retrieve the studied states first and this process in some way makes the other states less accessible. Therefore, the priority index for free recall of Postman \& Keppel (1967) was applied to the recall protocols to determine if output order of the two subsets was affected by prelearning. The percentage of high states occurring in the first half of each S's output was determined and subtracted from the number expected on the basis of a chance recall order. The mean value of this index, stated as a percentage of the possible difference, was 16.2 for Group PH, 8.2 for Group C, and 0.6 for Group PL. The trend is significant, $\mathrm{F}(2,79)=13.07, \mathrm{p}<.01$. In short studying the high subset produces a modest enhancement of output dominance for the high states, while studying the low subset produces an equal, but opposite, effect. In the light 
Table 1

Mean Number of States Recalled and Standard Deviations: Experiment 1

\begin{tabular}{|c|c|c|c|}
\hline \multirow{2}{*}{$\begin{array}{l}\text { Recall } \\
\text { Subset }\end{array}$} & \multicolumn{3}{|c|}{ Group } \\
\hline & $\mathrm{PH}$ & PL & $\mathrm{C}$ \\
\hline \multicolumn{4}{|l|}{ High } \\
\hline Mean & 24.04 & $21.41 *$ & $22.70^{*}$ \\
\hline $\mathrm{SD}$ & 1.22 & 2.72 & 1.75 \\
\hline \multicolumn{4}{|l|}{ Low } \\
\hline Mean & $15.73^{*}$ & 20.86 & $17.26^{*}$ \\
\hline $\mathrm{SD}$ & 4.55 & 2.92 & 3.91 \\
\hline
\end{tabular}

*.onlist stotes

of the data of Experiment 1 on both recall and output order, an attempt was made in Experiment 2 to amplify the differences found in recall of nonlist states by requiring experimental $\mathrm{Ss}$ to first attempt the recall of the subset they had studied and then attempt recall of the remaining states.

\section{EXPERIMENT 2}

\section{Method}

The materials used were those of Experiment 1, and the procedure was identical up to the point of the recall test. The test phase differed from Experiment 1 as follows: Ss in the two experimental groups were told to first output the 25 states they had studied before attempting to recall the other 25 states. Their instructions were "write down in any order you wish the names of the 25 states that you have just studied. When you have recalled as many as you can, draw a line and under it write the names of the other 25 states. In other words, your task is simply to write down the names of as many of the 50 states of the union as possible, writing the states you studied first." After $3 \mathrm{~min}$ of recall, if the $\mathrm{S}$ had not yet drawn the line and started recall of the nonlist states, he was told to start on them and that he had $3 \mathrm{~min}$ left. Those Ss who had already begun recall of the nonlist states (the great majority) and control Ss were simply told after $3 \mathrm{~min}$ that they had $3 \mathrm{~min}$ left to recall. Experimental Ss were also told that they could go back and write down the names of states they had studied if they thought of them after they had started on the nonlist states. All Ss were allowed a total of $6 \mathrm{~min}$ of recall. They were not asked to underline states after each minute of elapsed time in this study.

The Ss were drawn from the same source as those in Experiment 1 and were assigned randomly to the three groups until there were at least $12 \mathrm{Ss}$ per group. There were 12 Ss in Group PH, 14 Ss in Group PL, and 14 Ss in Group C. All Ss were tested individually.

\section{Results}

Table 2 shows the recall data of Experiment 2 arranged as in Tabie 1. All states recalled are included in the scoring. (Five studied states were listed as nonlist states by Group PH, while there were eight such errors by Group PL. Only one nonlist state was given as a studied state.)

Critical comparisons of nonlist states, as in Experiment 1, show significantly higher recall for both experimental groups than for the same subset in the control group. For Group PL vs Group C, comparison of the high subsets gives $t(26)=4.12$, $\mathrm{p}<.01$; for Group PH vs Group C, comparison of the low subsets gives $t(24)=2.18, \quad p<.05 . \quad$ A 2 by 2 analysis of variance treating the four entries as independent and adding two statistical Ss to Group PH gives significant main effects $(p<.01)$ for both study condition and recall subset with no interaction $(F<1)$.

$$
\text { DISCUSSION }
$$

The results of both experiments in general support the findings of Brown (1968) that the accessibility of one part of a larger set may be decreased when the accessibility of the other part is increased by prior exposure. The normative strength of the subset being recalled (or studied, since they are confounded) seems to be of little importance, at least with states. It may be fruitful to regard the John Brown effect as a special instance of retroactive inhibition in which the first stage, original learning, is assumed to have occurred preexperimentally, while the second stage, interpolated learning, has the special property of drawing material from the pool of items learned in the first stage.

To have implicated output interference as the process underlying the observed effect, as we have done here, is to leave much unsaid, it should be emphasized. Brown pointed out at least two different kinds of output
Table 2

Mean Number of States Recalled and Standard Deviations: Experiment 2

\begin{tabular}{lccc}
\hline & \multicolumn{3}{c}{ Group } \\
\cline { 3 - 4 } Recall & PH & PL & C \\
Subset & & & \\
\hline High & 24.00 & $20.71^{*}$ & $23.64^{*}$ \\
Hean & 1.15 & 1.86 & 1.78 \\
SD & & & \\
Low & & & \\
Hean & $14.92^{*}$ & 22.64 & $18.29^{*}$ \\
SD & 4.50 & 2.51 & 3.15 \\
\hline
\end{tabular}

*Nonlist states

interference which are theoretically possible. One mechanism goes as follows: "Successive retrievals from memory in free recall are somewhat analogous to sampling with replacement. This will mean that items which have already been retrieved are liable to occur as implicit intrusions while the $S$ is attempting to retrieve additional items, especially if the successful retrieval of an item still further increases its strength [p. 42, 1969]." We may complete this thought by suggesting that these implicit intrusions may serve to activate a termination rule by $S$ which functions to end active search. An alternative explanation offered by Brown is that preferred strategies for recalling the 50 states (for example, from east to west) may be disrupted by studying a subset. Neither hypothesis can be disconfirmed on the basis of the present data.

\section{REFERENCES}

BROWN. J, Reciprocal facilitation and impaiment of free recall. Psychonomic Science, 1968, 10, 41-42.

POSTMAN, L., \& KEPPEL, G. Retroactive inhibition in free recall. Joumal of Experimental Psychology, 1967, 74, 203-211.

WINER, B. J. Statistical principles in experimental design. New York: McGraw-Hill, 1962.

1. Two points about the analysis of variance should be clarified. First. because of unequal cell frequencies, seven statistical Ss were assigned the mean recall value of their groups and added in order to bring the $\mathrm{N}$ for each cell to 29 . However. degrees of freedom were computed on the actual number of $S$ s tested. Second, the Fs reported in both experiments may be regarded as conservative. since any correlation between the two sets of data from the control Ss has not been subtracted from the error term (Winer, 1963. pp. 42-43). 